\title{
Fluid Analog in Figh Energy Collisions
}

\author{
by \\ K. Tanaka $a^{x}$ \\ and \\ Y. Ueda $x \mathrm{x}$ )
}

x) Institute of Theoretical Physics, Göteborg, Sweden, and Dopartment of Physics, Ohio State University, Columbus, Ohio 43210, USA

$x x$ ) Institute of Theoretical Physics, Göteborg Sweden

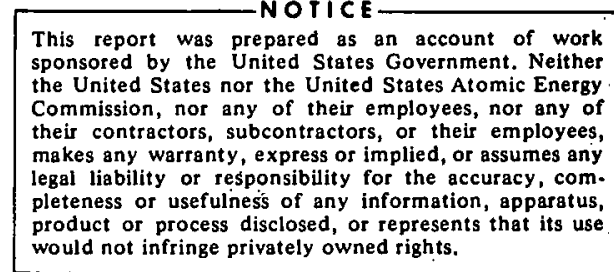

*

Work supperted in part by the U.S. Atomi: Erergy Comission under contract $\operatorname{AT}(i 1-1)-1545$

Institute of Theoretical Physics

Fack

S-402 20 GÖTERORG 5

Sweder. 


\section{DISCLAIMER}

This report was prepared as an account of work sponsored by an agency of the United States Government. Neither the United States Government nor any agency Thereof, nor any of their employees, makes any warranty, express or implied, or assumes any legal liability or responsibility for the accuracy, completeness, or usefulness of any information, apparatus, product, or process disclosed, or represents that its use would not infringe privately owned rights. Reference herein to any specific commercial product, process, or service by trade name, trademark, manufacturer, or otherwise does not necessarily constitute or imply its endorsement, recommendation, or favoring by the United States Government or any agency thereof. The views and opinions of authors expressed herein do not necessarily state or reflect those of the United States Government or any agency thereof. 


\section{DISCLAIMER}

Portions of this document may be illegible in electronic image products. Images are produced from the best available original document. 
Abstract:

We have used the generating functions which represent the $p p$ data from 50 to $303 \mathrm{GeV} / \mathrm{c}$ as analog grand partition functions and explored notions of analog pressure, specific volume, equation of state ana critical point. We also discuss $\ell$-plane singularities in this connection. 


\section{Introducticn}

There is recent interest in the possibility that tha multiplicity data on $\mathrm{p} \mathrm{p}$ collisions above $100 \mathrm{GeV} / \mathrm{c}$ may be interpreted in, terms of a language familiar in statistical mechanics ${ }^{1)}$. This idea is reinforced when we note the similarity between the gener iting function $Q(z, Y)$ in particle production and the grand partition function in statistical mechanics. Both form a medium from which one can derive physical quantities.

The generating furction is defined by the equation

$$
Q(Z ; Y)=\sum_{n}^{\infty} \sigma_{n}(Y) Z^{n} / \sigma_{m}(Y)
$$

where $\sigma_{n}(Y)$ is the topological cross section for $n$ charged particles, $\sigma_{i n}\left(Y^{\prime}\right)=\sum_{n} \sigma_{n}(Y)$ is the inelastic corss section. A variable $Y$ is defired by $Y=\ln \left(S / S_{0}\right)$ where $S^{1 / 2}$ is the center or mass energy of the $\mathrm{p}$ p system and $\mathrm{z}$ is à parameter.

A particular choice of the parameter, $z=1$, fixes the normalization of $Q$.

$$
Q(1, Y)=\sum_{n} \sigma_{n} / \sigma_{m}=1 .
$$

The first derivative of $z$ at $z=1$ yields the average multiplicity $\langle n\rangle$ :

$$
\left.\frac{d Q(Z, Y)}{d Z}\right|_{Z=1}=\langle n\rangle,
$$

where

$$
\langle n\rangle=\sum_{n} n \sigma_{n} / \sigma_{\text {in }} .
$$


Similarly, higher order derivatives at $z=1$ give the binomial moments; for example.

$$
\left.\frac{d^{2} Q(Z, Y)}{d z^{2}}\right|_{z=1}=\langle n(\dot{n}-1)\rangle \text {, }
$$

where

$$
\langle n(n-1)\rangle=\sum_{n} n(n-1) \sigma_{n} / \sigma_{\text {in }} .
$$

Although $z$ is merely a parameter here, it may be interpreted as the square of the coupling constant in some Regge-type modeis. In statistical mechanics $\sigma_{n}$ corresponds to the canonical partition function and $z$ is the fugacity. The variable $Y$ plays the role of volume.

In particular we are interested in studying the viability of such analog variabies with the aid of the generating functions which represent the data on high energy $p$ p collisions from 50 to $303 \mathrm{GeV} / \mathrm{c}$. In view of the fact that the analog grand partition $Q(z, Y)$ is obtained in a closed form, it is possible to make definite statements based on the experimental facts. At these energies, there is some theoretical justification for Koba, Nielsen, Olesen (KNO) scaling. 2). There is recent interest in the fluid analog 3) 4). The analog pressure $P$, the single particle density $\rho$ and the specific volume $v$ are defined in the following as a function of. $z$ and $Y$.

$$
\begin{aligned}
P(Z, Y) & =\frac{1}{Y} \ln _{n} Q(Z, Y) \\
P(Z, Y) & =v^{-1}(Z, Y) \\
& =\frac{1}{Y} Z \frac{\partial}{\partial Z}[\ln Q(Z, Y)]
\end{aligned}
$$


In statistical mechanics, the limit $\mathrm{Y} \rightarrow \infty$ is taken. Here $\mathrm{Y}$ is considered to be arbitrary large and kept fixed. The explicit Y dependence in the physical quantities may sometimes be suppressed. For a fixed $Y$, we can construct an equation of state, which is the equation expressing $P(z, Y)$ as a function $v(z, Y)$, by eliminating the explicit $\mathrm{z}$ dependence. Then we consider the limit $\mathrm{Y} \rightarrow \infty$ in order to discuss the possible existence of critical points.

It is interesting to examine whether the hadronic fluid has a critical point which is a singularity or discontinuity in the equation of state. According to thermodynamics $P$ is a decreasing function of v. i.e.

$$
\frac{\partial P}{\partial v}<0
$$

At the critical point we must have

$$
\frac{\partial P}{\partial v}=0
$$

Furthermore, the equilibrium of a state with $\frac{\partial P}{\partial v}=0$ requires the second derivative to vanish simurtaneously 5 )

$$
\frac{\partial^{2} p}{\partial v^{2}}=0
$$

In our hadronic case $\quad \frac{\partial P}{\partial v}<0$ is satisfied in the models considered here. We shall examine the possible existence of critical points by constructing explicitly ths eçuation of state.

A more direct approach is to investigate the analytic property of $Q(z, Y)$ when $z$ is regarded as a complex variable 5) (3) This 
approsch becomes particularly powerful, when the form of the generating function is compactly know such as those given in section 2.

Present empirical fits do not seem to support the occurrence of a. phase transition in the hadronic fluid.

We also investigate the singularity structure in the complex $l$-plane, based on a fluid analog. Existence of a cut contribution in the Regge theory can be understood as an explicit (or finite) $Y$ dependence of analog pressure.

In section 2 we take up the exponential model of $\sigma_{n}$ and in section 3 the gaussian model of $\sigma_{n}$. In section 4 we briefly discuss the equation of state. In section 5 we discuss singularitites in the complex $\ell$-plane. 


\section{Exponential model of $\sigma_{n}$.}

According to slattery $\left.{ }^{7}\right)$ the following kro scaling function $\psi(n \mid\langle n\rangle)$ can fit the high energy $p$ p collision data:

$$
\sigma_{n}=\sigma_{\text {in }} \frac{1}{\langle n\rangle} \psi(n /\langle n\rangle) \text {, }
$$

where

$$
\psi(x)=\sum_{L=1,3,5,7} N_{L} x^{L} e^{-a x} .
$$

- Here, $\sigma_{\text {in }}$ is the inelastic cross section $\sigma_{i n}=\sum_{n} \sigma_{n}, x=n\langle\langle n\rangle$ and $\langle n\rangle$ is the average charged multiplicity. Lack of even values of $L$ come from the experimental data. Numerically, $a=3.04$. $\mathrm{N}_{1}=3.79 \mathrm{~N}_{3}=33.7 \quad \mathrm{~N}_{5}=-6.64^{\circ}$ and $\mathrm{N}_{7}=0.332$. We note that experimentaliy $\langle n\rangle$ is a runction of $Y$ and increases as $Y \rightarrow \infty$. Construction of the generating function and the relation with correlation function for the above empirical fit are given in reference 8 ): In order to simplify our discussion we first present a case of $L=0$ and will consider a realistic case later.

For $L=0$, we have

$$
\sigma_{n}(Y)=\operatorname{\sigma in} \frac{N_{0}}{\langle n\rangle} e^{-a n /\langle n\rangle} .
$$

Normalization of $\sigma_{n}$ determines No is

$$
N_{0}=\langle n\rangle\left(1-e^{-a\langle n\rangle}\right)
$$

The generating function $Q(z, Y)$ is ootained from eqs. (1.1), (2.2) and (2.3)

$$
Q(Z, Y)=\left(1-e^{-a \mid\langle n\rangle}\right) /\left(1-\neq e^{-a\langle i n\rangle}\right)
$$


Parane:ter a can be determined from eq: (1.3) as

$$
a=\langle n\rangle \ln \left(1+\frac{1}{\langle n\rangle}\right) .
$$

Pressure and specific voluine are calculated with the aid of (1.2), $(1.3)(2.4)$ and $(2.5)$ to be

$$
\begin{aligned}
& P(Z, Y)=-\frac{1}{Y} \ln [1+\langle n\rangle(1-Z)], \\
& v(Z, Y)=\left(\frac{Y}{Z\langle n\rangle}\right)[1+(1-Z)\langle n\rangle] .
\end{aligned}
$$

The elimination of $z$ from eqs. (2.6) and (2.7) yields the equation of state

$$
P(Z, Y)=\frac{1}{Y} \ln \left[\left(1+\frac{Y}{v(Z, Y)}\right) \cdot(1+\langle n\rangle)\right] .
$$

One notes from eq. (2.7) that $v(Z, Y)$ requires $\frac{1}{\langle\eta\rangle}+1>z \geq 0$ which is also the condition for the convergence of the original series $Q(z, Y)$, i.e. $\left|Z e^{-a /\langle n\rangle}\right|\langle|$ This means that the analog specific volume must be non negative for the grand partition function $Q(z, Y)$ to exist.

It follows from eqs. $(2.6)$ and $(2.7)$ that for the cose $z=1$,

$$
\begin{aligned}
& P(1, Y)=0 \\
& v\left(1, Y^{\prime}\right)=Y /\langle n\rangle .
\end{aligned}
$$

The value of the pressure dejends on the normalization of $Q(Z, Y)^{3}$. If one replaces the $\sigma_{\text {in }}$ in eq. $(2.1)$ by some function $\sigma_{c}(y)$, one 
obtains $P^{\prime}(z)=P(z)+P_{i}(Y)$ where $\quad P_{0}(Y)=\ln \left[\sigma_{\text {in }} / \sigma_{0}\right] / Y$.

Note that. $P O$ is indepencent of $z$ and $V^{\prime}(z)=V(z)$, so that the efrect

on the equation of the state is to replace $\mathcal{P}(z)$ by $P^{\prime}(z)-P_{0}(Y)$.

The different forms of $Q(z, Y)$ leads to a shift in the $P(z)$.

We obtain from eq. (2.8)

$$
\begin{aligned}
& \frac{\partial P(Z, Y)}{\partial v(Z, Y)}=-\left[v^{2}(Z, Y)+v(Z, Y) Y\right]^{-1}<0, \\
& \frac{\partial^{2} P(Z, Y)}{\partial v^{2}(Z, Y)}=\frac{2 v(Z, Y)+Y}{\left[v^{2}(Z, Y)+v(Z, Y) Y\right]^{2}}>0,
\end{aligned}
$$

which do not satisfy the conditions for a critical state when $Y$ is finite. In order to find the critical points we have to take the limit $Y \rightarrow \infty$. Experimentally; the average charged multiplicity seems to increase linearly with $Y$, although tinere are other possibilities. Here we accept $\langle\eta\rangle \propto \gamma$ as $Y \rightarrow \infty$. When we take the limit $Y \rightarrow \infty$ maintaining $\ddot{v}(1)=Y /\langle n\rangle$ a constant, we find a rather trivial result .

$$
\begin{aligned}
& p(z, \infty)=0 \\
& \frac{\partial p(z, \infty)}{\partial v(z, \infty)}=\frac{\partial^{2} p(z, \infty)}{\partial v^{2}(z, \infty)}=0
\end{aligned}
$$

, meaning no critical point.

We can understand tinis situation in a slightly different way. Let us regara $Q(Z, Y)$, given in eq. (2.4) and reproduced here, as a function of the compiex variable $z: 5), 6$ ): 


$$
Q(Z, Y)=\frac{i-e^{-a \mid\langle n\rangle}}{1-Z e^{-a \mid\langle n\rangle}} .
$$

As is discussed earlier, the positivity of the specific volume $v(Z, Y)$ requires

$$
\left|z e^{-a\langle n\rangle}\right|<i
$$

For the region where eq. (2.11) is satisfied, eq. (2.4) is analytic, and so is $P(z, Y)$ and $V(Z, Y)$. Since our argument is independent of the magnitudes of $Y$ we can let $Y \rightarrow \infty$.

For $|z|<1, P(z, \infty)$ and $v(z, \infty)$ are analytic. Thus no singularity or discontinuity can appear in the equation of state, which means nonexistence of critical points.

Next we discuss the case with an arbitrary L:

$$
\sigma_{n}=\sigma_{i n} \frac{N_{L}}{\langle n\rangle}\left(\frac{n}{\langle n\rangle}\right)^{L} e^{-\alpha n\langle n\rangle}
$$

The generating function can be similarly constructed

8)

$$
\begin{aligned}
& Q^{(L)}(Z, Y)=\frac{\partial^{L}}{\partial\left(-\frac{a}{\langle n\rangle}\right)^{2}}\left(1-Z e^{-a\langle n\rangle}\right)^{-1} / \frac{\partial^{L}}{\partial\left(-\frac{a}{\langle n\rangle}\right)^{L}}\left(1-e^{-a /\langle n\rangle}\right)^{-1} \\
& \text { with } Q^{(L)}(1, Y)=1
\end{aligned}
$$

Here a superscript specifies the contribution from a particular I.

Taking derivatives explicitly, we find

$$
Q^{(L)}(Z, Y)=Z^{L}\left[Q^{(0)}(Z, Y)\right]^{L+1}
$$

where

$$
Q^{(0)}(z, Y)=\frac{\left(1-z e^{-a\langle k n\rangle}\right)^{-1}}{\left(1-e^{-a k n\rangle}\right)^{-1}}
$$


Pressure and specific volume can be similarly calculated

$$
E(Z, Y)=\frac{L}{Y} \ln Z+(L+1) P^{\prime}\left(q^{\prime} Z, Y\right)
$$

$$
\frac{1}{v(Z, Y)}=\frac{L}{Y}+(L+1) \frac{1}{v^{(0)}(Z, Y)} \text {. }
$$

where $p^{(0)}(Z, Y)$ and $v^{0}(Z, Y)$ are pressure and specific volume for $L=0$ :

$$
\begin{aligned}
& P^{(0)}(z, Y)=\frac{1}{Y} \ln R^{(0)}(z, y), \\
& \frac{1}{v^{(0)}(z y)}=\frac{z}{Y} \frac{e^{-a \mid k n)}}{1-z e^{-a k n\rangle}}
\end{aligned}
$$

where parameter a is determined from

$$
e^{-a\langle n\rangle}=\frac{\langle n\rangle-1}{\langle n\rangle+1}
$$

that it is

An essential feature of the arbitrary $L$ case is the same as the $L=0$ case. This is particularly true if we can neglect the first term of ens. (2.14) and (2.15).

We now construct a generating function corresponding to the empirical fit of eq. (2.1):

$$
\begin{aligned}
Q(z, Y) & =\sum_{0 d d L}^{T} N_{L} Q^{(L)}(z, y) / \sum_{0 d d L}^{T} N_{L} \quad\left(Q^{(L)}(1, y)=1\right) \\
& =\sum_{0 d d L}^{7} N_{L} Z^{L}\left[Q^{(0)}(z, y)\right]^{L+1} / \sum_{0 d d L}^{T} N_{L} \cdot(2.19)
\end{aligned}
$$

Parameter a is now determined by

$$
e^{-\frac{a}{\langle n\rangle}}=\frac{\langle n\rangle-\langle L\rangle}{1+\langle n\rangle}
$$


where

$$
\left\langle L=\sum_{\text {odd } L}^{7} N_{L} L / \sum_{\text {od di }}^{7} N_{L}\right.
$$

Pressure and specific volume can in similarly calculated.

$$
\begin{aligned}
& P(Z, Y)=\frac{1}{Y}\left(\ln \left[\sum_{0 d d L}^{T} N_{L} Z^{L}\left(Q^{(0)}(z, Y)\right)^{L+1}\right]-\ln \left[\sum_{0, d}^{T} N_{L}\right]\right)_{(2.21)}
\end{aligned}
$$

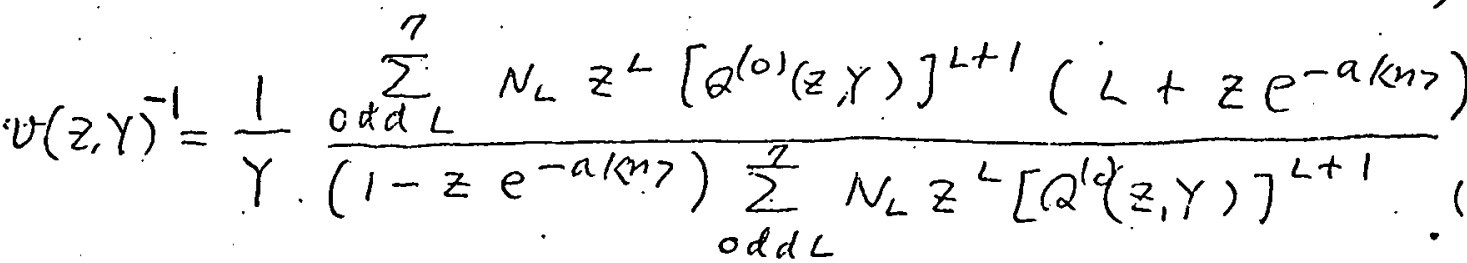

By eliminating $z$ in $P(z, Y)$ and $V(z, Y)$ we can construct ar equation of state for fixed. $Y$. We note that the valid range of $z$ is the same as that for the $I=0$ case, ie.

$$
0 \leq z \leq 1+\frac{1}{\langle n\rangle}
$$

This is because the $I \neq 0$ case is constructed from the $L=O$ case by taking derivatives.

We will discuss the $P-v$ diagram in section 4 . 
11.

3. Gaussian model of $\sigma_{n}$.

An alternative form for the KNO scaling function has been proposed by Weisberg ${ }^{9)}$ in order to fit the pp collision data:

$$
\sigma_{n}=\sigma_{m} N_{m} \frac{n^{m}}{\langle n\rangle^{m+1}} e^{-\alpha n^{2} /\langle n\rangle^{2}}
$$

where

$$
\begin{aligned}
& N_{m}=4 \alpha^{(m+1) / 2} / \Gamma[(m+1) / 2], \\
& \alpha^{1 / 2}=\Gamma[(m+2) / 2] / \Gamma[(m+1) / 2] .
\end{aligned}
$$

$(3.3)$

Here $\mathrm{n}$ is an even integer and $\mathrm{m}$ is an adjustable positive parameter (experimentally $\mathrm{m}=1.215$ ).

After the summation in the generating function is approximated by an integral, we have

$$
Q(Z, Y)=N_{m}\langle n\rangle^{-(m+1)} 2^{m} \int_{0}^{\infty} d t t^{m} Z^{2 t} e^{-\frac{4 \alpha}{\langle n\rangle^{2}} t^{2}}
$$

$$
\begin{aligned}
& Q(Z, Y)=\exp \left[\langle n\rangle^{2}(\ln z)^{2} / 8 \alpha\right] x \\
& \dot{U}\left(m+\frac{1}{2},-\langle n\rangle \ln z /(2 \alpha)^{1 / 2}\right) / U\left(m+\frac{1}{2}, 0\right)
\end{aligned}
$$

where $U$ is a parabolic cylinder function. 10)

We obtain from eqs. $(1.2),(1.3)$ and (3.5)

$$
\begin{aligned}
& P(Z, Y)=\frac{(n)^{2}(\ln Z)^{2}}{8 \alpha Y}+\frac{1}{Y} \ln \left[u\left(m+\frac{1}{2},-(n) \ln Z /(2 \alpha)^{\frac{1}{2}}\right) / U\left(m+\frac{1}{2}, 0\right)\right],
\end{aligned}
$$

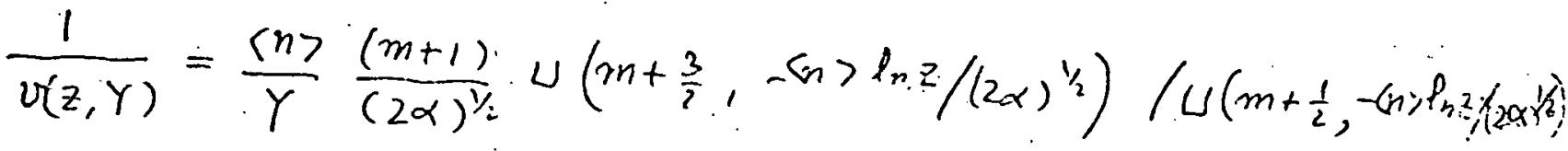


where the following recurrence relation for $U(a, x)$ was used:

$$
\frac{d v(a, x)}{d x}=-\frac{1}{2} x \omega(a, x)-\left(a+\frac{1}{2}\right) v(a+1, x) \cdot \text { (3.8) }
$$

It is appropriate to consider the three $\operatorname{cases}^{\circ} \mathrm{z}=1, \mathrm{z}>1$ and $z<1$ separately

In the case $z=1$, we have

$$
\begin{aligned}
& P(1, Y)=0 \\
& \vdots(1, Y)=Y\langle\langle n\rangle .
\end{aligned}
$$

In the case $z>1$, when $\left.\frac{\langle n\rangle|\ln z|}{(2 \alpha)^{1 / 2}}\right\rangle m+\frac{1}{2}$ is satisfied, we have the following asymptotic forms

$$
\begin{gathered}
P(Z, Y) \approx \frac{1}{Y} \cdot\left\{\ln \left[2^{\frac{m+2}{2}} \frac{\Gamma\left(\frac{m+2}{2}\right)}{\Gamma(m+1)}\right]+m \ln \left[(n\rangle \frac{\ln z}{(2 \alpha)^{1 / 2}}\right]\right. \\
\left.+\frac{\langle n\rangle^{2}(\ln z)^{2}}{4 \alpha}\right\}, \\
v(z, Y) \approx 2 \propto Y /\left(\langle n\rangle^{2} \ln z\right)
\end{gathered}
$$

The equation of state is -

$$
\begin{aligned}
P(z, y)=\frac{1}{y}\{\ln [ & \left.\frac{2^{\frac{m+2}{2}} \Gamma\left(\frac{m+2}{2}\right)}{\Gamma(m+1)}\right]+m \ln \left[\frac{(2 \alpha)^{\frac{1}{2}} v(1, y)}{v(z, y)}\right] \\
& \left.+\frac{v^{2}(1, y) \alpha}{v^{2}(z, y)}\right\}
\end{aligned}
$$

In the case $z<1$, when $\frac{\langle n\rangle\left|P_{n} z\right|}{(2 \alpha)^{1 / 2}} \gg m+\frac{1}{2}$ is satisfied, we have the following asymptotic forms

$$
\begin{aligned}
& P(Z, Y) \approx-\frac{(m+1)}{Y} \ln \left[-\langle n\rangle \ln Z /\left((2 \alpha)^{1 / 2}\right]-\frac{1}{\gamma} \ln \left[u\left(m+\frac{1}{2}, 0\right)\right](3.14)\right. \\
& v(Z, Y) \approx-Y \ln Z /(m+1) .
\end{aligned}
$$


13.

The equation of state is

$$
P(Z, Y) \approx-\frac{1}{Y}\left(m+1 ; \ln \left[\frac{(m+1) v(z, Y)}{(2 \alpha)^{1 / 2} v(1, Y)}\right]-\frac{1}{Y} \ln \left[U\left(m+\frac{1}{2}, 0\right)\right] 3.16\right) \text {. }
$$

The above condition $\left.\frac{\left(n>\mid P_{n} z\right)}{(2 \alpha)^{1 /}}\right\rangle m+\frac{1}{2}$ ill be net as $\langle n\rangle \rightarrow \infty$ as far as $\ln z \neq 0$.

In practice, however, $\langle n\rangle$ is finite and it is sometime necessary, specially for small In $z$, to use the following expansion

$$
\begin{aligned}
P(Z, Y) & \left.=\frac{1}{Y}\left[\left(m+\frac{1}{2}\right)^{1 / 2} y+\left(\frac{1}{4}-\frac{1}{16\left(m+\frac{1}{2}\right)}\right) y^{2}+\frac{1}{24} \frac{1}{\left(m+\frac{1}{2}\right)^{1 / 2}} y^{3}+\cdots\right] .17\right) \\
U(z, Y) & =\frac{Y}{\langle n\rangle} \frac{(2 \alpha)^{1 / 2}}{(m+1)} \frac{U\left(m+\frac{1}{2}, 0\right)}{U\left(m+\frac{3}{2}, 0\right)} \exp \left[\left\{\left(m+\frac{1}{2}\right)^{1 / 2}-\left(m+\frac{3}{2}\right)^{1 / 2}\right\} y\right. \\
& +\frac{1}{16}\left\{-\left(\frac{1}{\left(m+\frac{1}{2}\right)}+\frac{1}{\left(m+\frac{3}{2}\right)}\right\} y^{2}+\frac{1}{24}\left\{\frac{1}{\left(m+\frac{1}{2}\right)^{1 / 2}}-\frac{1}{\left(m+\frac{3}{2}\right)^{1 / 2}}\right\}(3.18)\right.
\end{aligned}
$$

where $y=\frac{\langle n\rangle \ln z}{(2 \alpha){ }^{1 / 2}}$.

These expressions are valid for $m+\frac{1}{2} \gg y^{2}$ 


\section{Discussions}

We now evaluate $P(z, Y)$ at the maximally available energy $303 \mathrm{GeV}$.

For numerical aralysis, we use $Y=\ln s-1.53$ ).

The experimental value of $\langle n\rangle$ is $(7)$

$$
\langle n\rangle=8.86 \pm 0.15^{\circ}
$$

We find that the exponential model and the gaussian model yield almost identical results. (See Figs. 1 and 2.)

The main difference appears in the range of validity of $z$. In the exponential model $\mathrm{z}$ must be restricted to

$$
0 \leq \mathrm{z} \leq 1.12
$$

while the gaussian model is not required to have any restriction on $\mathrm{z}$ :

$$
0 \leq \mathrm{z} \leq \infty
$$

The $\mathrm{p}-\boldsymbol{v}$ diagram at $303 \mathrm{GeV}$ is plotted in Fig. 3.

We observe in Fig. 3 that $\frac{\partial P}{\partial v}<0$ and that there is no point at this energy satisfying $\frac{\partial P}{\partial v}=0$. We would like to add a remark about $z=1$ in connection with a phase transition. In the limit $Y \rightarrow \infty$, physical quantities such as $V(z, Y)$ could become singular at $z=1$. However, $z=1$ can not be a critical point, since in the limit $Y \rightarrow \infty$ the allowed region for $z$ is shrunk to

$$
0 \leq z<1
$$

wrich is the range of convergence of series $Q(z, Y)$ in both models.

Thus the present empirical fits do not seem to support the occurrence of a phase transition. 
When there is a simple Regge pole exchange in multiperipheral models, one has short range correlations whereas eqs. (2.1) and (3.1) lead to long range correlations i.e. the two particle correlation function $f_{2} \sim\langle n\rangle^{2}$.

Equations (5.4) and (5.5) indicate the complex exchange process.

In conclusion, if pressure $P(z, Y)$ depended only on $z$, such as $P(z, \infty)$, then ve should have obtained Regge poles ratier tahn cuts.

Importance of cut contributions in Regge phenomenology may indicate that $Y$ dependence of the pressure can not be ignored. 


\section{Singularitics in complex $l$ plane}

In uruer to understand the long range correlations which are present in the $p$ data as exemplified by the KNO scaling model, we are going to express our generating function $Q(z, Y)$ in the Regge language. Then the asymptotic energy dependence of $Q(z, Y)$ is controlled by a few singularities in the complex $\ell$ plane. According to our definition (1.4), we have

$$
Q(Z, Y)=e^{P Y}
$$

In order to study the $\ell$ dependence, we construct its Japlace transform defined by

$$
\begin{aligned}
Q(z, l) & \equiv \int_{0}^{\infty} d Y e^{-\ell Y} Q(z, Y) \\
& =\int_{0}^{\infty} d Y e^{-(l-P) Y}
\end{aligned}
$$

Since $P$ depends upon $Y$ also, $Q(z, Y)$ does not produce poles, but a cut.

Substituting eqs. (1.2) and 2.6) for the exponential $\sigma_{n}$ with $L=0$, for instance, we get

$$
Q(z, l)=\int_{0}^{\infty} d y \frac{e^{-y}}{\ell-[(z-1) y(v(1)]} .
$$

Equation (5.4) exnizits a cut in the $\ell$-plane for $z>1$ and $\operatorname{Re} \ell \geq 0$. In a similar nanner, fie get from $(1.2)$ and $(3.4)$ for the gaussian $\sigma_{n}$

$$
Q(z l)=\frac{1}{2^{\frac{m-1}{2}} 1\left(\frac{m+1}{2}\right)} \int_{0}^{\infty} d s \frac{s^{m} e^{-\frac{1}{2} s^{2}}}{l-s\left[\ln z / v(1)(2 \cdot \alpha)^{\frac{1}{2}}\right]}
$$

in which $Q(z, y)$ again exhibits a cut when $z>1, l \geq 0$. 
The authors would like to thank J. Nilsson and $\tilde{n}$. E. Eriksson for their hospitality at the Institute for Theoretical Physics, and to G.H. Thomas, K.E. Erjksson and A. Sjölander for interesting discussions. The authors enjoyed the stimulating atmosphere of the Weak Interaction Work Shop at Skövde, Sweden where ideas for the present work were formulated. 


\section{References}

(1) R.P. Feynman, Particle Physics (Irvine Conferences, 1971), A.I.P. Conference proceedings, No. 6; K.G. Wilson, Cornell Unj.versity Report, No. CLNS-131; 1970; J.I. Bjorken, in

"Particles and Fields", 1971 APS Rochester meeting proceedings.

(2) Z. Koba, H.B. Nielsen and P. Olesen, Nucl. Phys. 1340 (1972) 317.

(3) R.C. Arnold, ANL/HEP 7317 (1973); R.C. Arnold and G.H. Thomas, ANL/HEP 7257 (1973).

(4) M. Bander, Phys. Rev. Letters 30 (1973) 460; Phys.Rev. D6 (1.972) 164

T.D. Lee, Phys.Rev. D6 (1972) 3617.

(5) K. Huang, Statistical Mechanics (Wiley, 1963); L.D. Landau and E.M. Liftshitz, Statistical Physics (Addison-Wesley, Reading, Mass., 1958).

(6) C.N. Yang and T.D. Lee, Phys.Rev. 87 (1952) 404.

(7) P. Slattery, Phys.Rev. Letters 29 (1972) 1624; Phys.Rev. D7 (1973) 2073; P. Olesen, Phys. Letters 41B (1972) 602.

(8) G.J. Aubrecht, K. Tanaka and R. Torgerson, Ohio State University Report No. CoO-1545-125, 1973.

(9) H. Weisberg, University of Pennisylvania Report No: UR-409, 1972.

(10) M. A.bramowitz and. I.A. Stegren, Handbook of Mathematical Functions pg. $687 \mathrm{ft}$ : 


\section{Figure captions}

Fig. 1 Specific volume $v$ as a function of $\mathrm{z}$ at $\sqrt{\mathrm{s}}=303 \mathrm{GeV}$ - : Exponeritial model

- : Gaussian model

Fig. ? Pressure $P$ as a function of $z$ at $\sqrt{s}=303 \mathrm{GeV}$

- Exponential model

- : Gaussian model

Fig. 3 Pressure versus specific volume at $\sqrt{\mathrm{s}}=303 \mathrm{GeV}$

- Exponential model

- : Gaussian model 


\section{$=303$}

$P(z)$

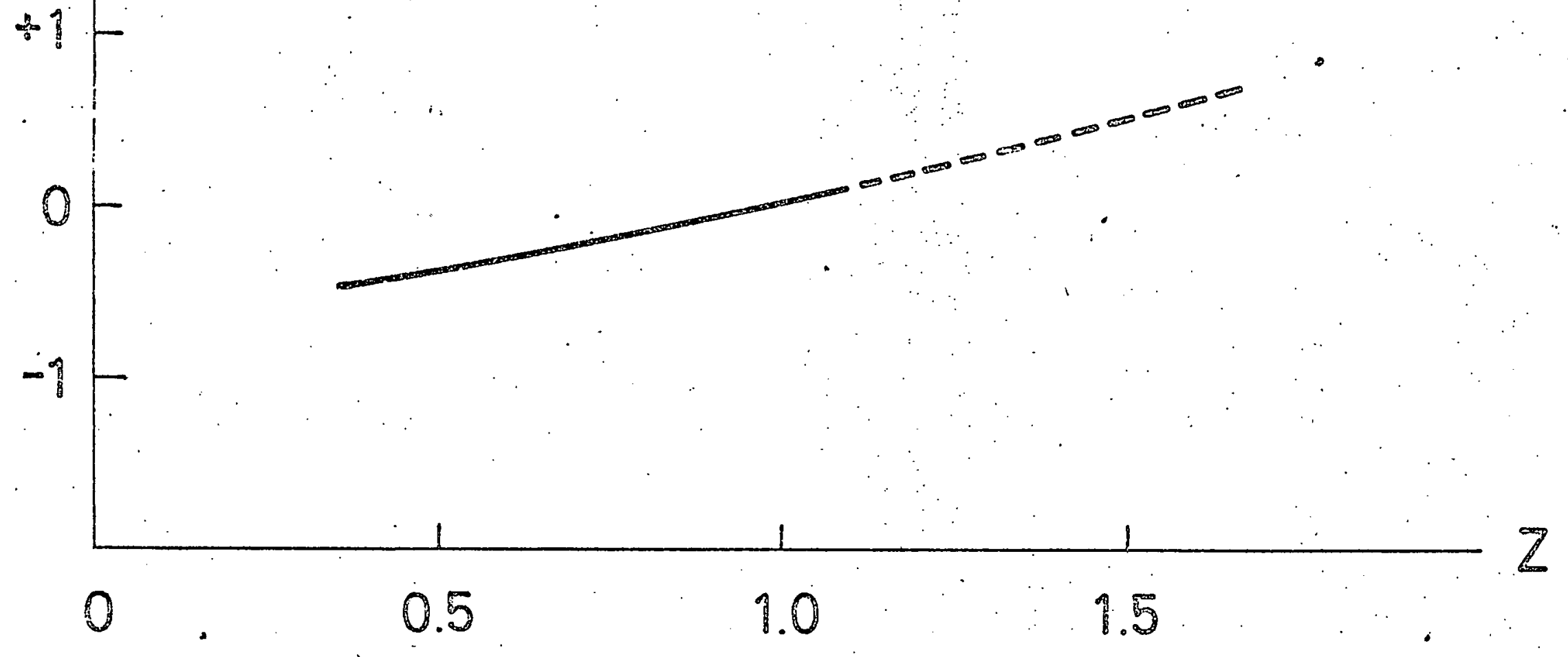




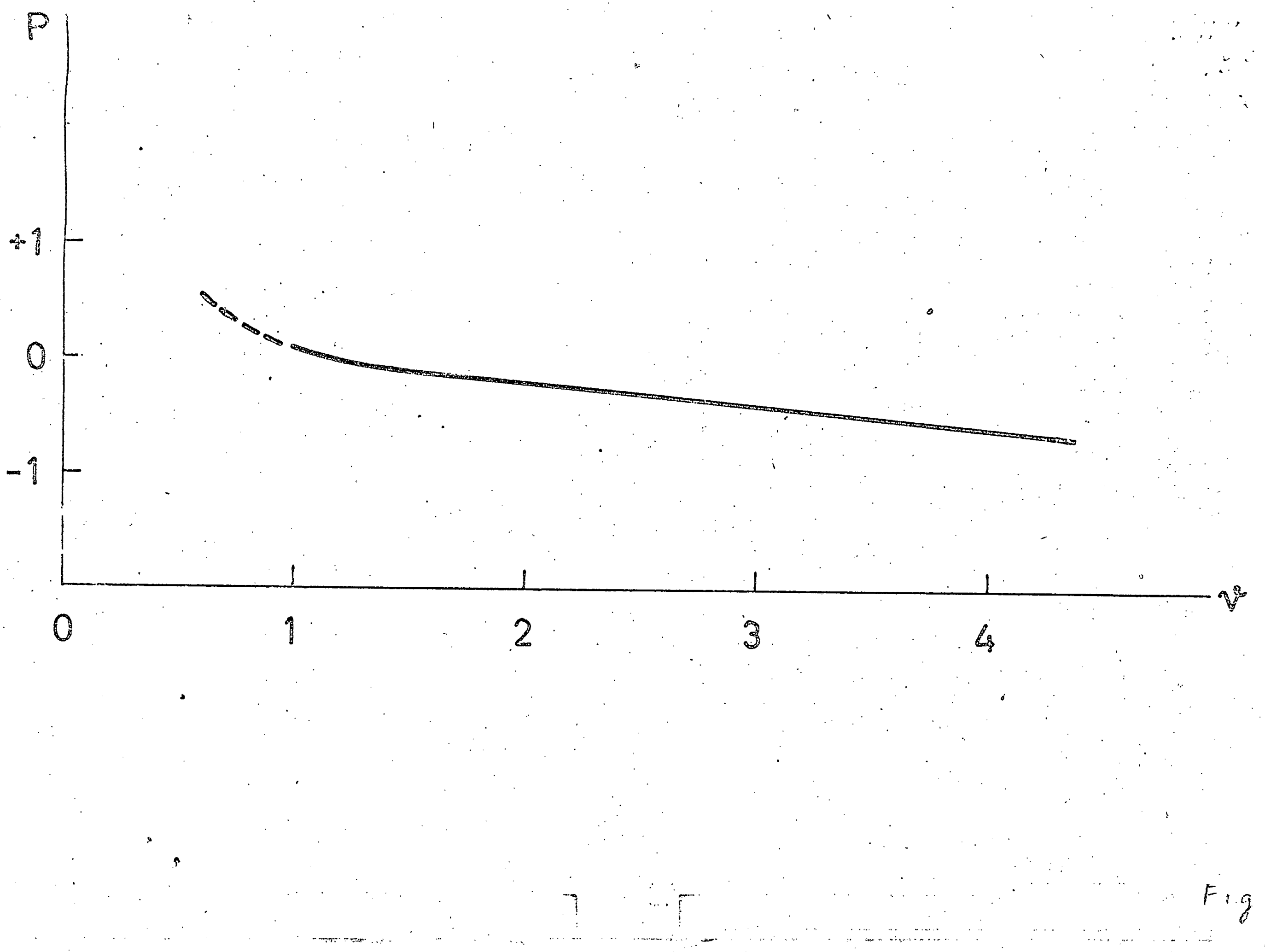

